\title{
Immunopathogenesis of Hepatic Brucellosis
}

\author{
Guillermo Hernán Giambartolomei and María Victoria Delpino* \\ Instituto de Inmunología, Genética y Metabolismo (INIGEM), Universidad de Buenos Aires, Buenos Aires (UBA), Consejo \\ Nacional de Investigaciones Científicas y Técnicas (CONICET), Buenos Aires, Argentina
}

OPEN ACCESS

Edited by:

Nesrin Ozoren,

Bogaziçi University, Turkey

Reviewed by:

Kai Yang,

Indiana University School of Medicine-Lafayette, United States Thorsten Maretzky,

The University of lowa, United States

${ }^{*}$ Correspondence: María Victoria Delpino

mdelpino@ffyb.uba.ar

Specialty section: This article was submitted to Microbes and Innate Immunity, a section of the journal Frontiers in Cellular and Infection Microbiology

Received: 27 August 2019 Accepted: 28 November 2019 Published: 20 December 2019

Citation:

Giambartolomei GH and Delpino MV (2019) Immunopathogenesis of Hepatic Brucellosis.

Front. Cell. Infect. Microbiol. 9:423.

doi: 10.3389/fcimb.2019.00423
The hepatic immune system can induce rapid and controlled responses to pathogenic microorganisms and tumor cells. Accordingly, most of the microorganisms that reach the liver through the blood are eliminated. However, some of them, including Brucella spp., take advantage of the immunotolerant capacity of the liver to persist in the host. Brucella has a predilection for surviving in the reticuloendothelial system, with the liver being the largest organ of this system in the human body. Therefore, its involvement in brucellosis is practically invariable. In patients with active brucellosis, the liver is commonly affected, and the most frequent clinical manifestation is hepatosplenomegaly. The molecular mechanisms implicated in liver damage have been recently elucidated. It has been demonstrated how Brucella interacts with hepatocytes inducing its death by apoptosis. The inflammatory microenvironment and the direct effect of Brucella on hepatic stellate cells (HSC) induce their activation and turn these cells from its quiescent form to their fibrogenic phenotype. This HSC activation induced by Brucella infection relies on the presence of a functional type IV secretion system and the effector protein BPE005 through a mechanism involved in the activation of the autophagic pathway. Finally, the molecular mechanisms of liver brucellosis observed so far are shedding light on how the interaction of Brucella with liver cells may play an important role in the discovery of new targets to control the infection. In this review, we report the current understanding of the interaction between liver structural cells and immune system cells during Brucella infection.

\section{Keywords: liver, fibrosis, inflamación, hepatocite, stellate cells (Ito cells)}

\section{BRUCELLOSIS}

Brucellosis is a common zoonotic disease distributed around the world. The disease is rare in industrialized countries because of the routine screening of domestic livestock and animal vaccination program (Pappas et al., 2006; Mancini et al., 2014). Recently, case studies and review of published cases have been carried out to determine the prevalence of brucellosis in endemic regions. However, it should be borne in mind that because many areas with endemic brucellosis have poor infrastructure, it is likely that the incidence of the disease is underestimated. There are a reported 500,000 incident cases of human brucellosis per year. However, true incidence is estimated to be 5,000,000 to 12,500,000 cases annually (Godfroid et al., 2013; Hull and Schumaker, 2018). The clinical disease is common in the Middle East, Asia, Africa, South and Central America, the Mediterranean Basin, and the Caribbean (Pappas et al., 2006; Mancini et al., 2014; Cross et al., 2019). 
At present, 12 species of Brucella genus have been described, each of which has its natural host, including domestic and farm animals, as well as wild animals, such as camels, bison, foxes, cetaceans, among others. Brucella is an expanding genus, and the most recent species were isolated from amphibians (Eisenberg et al., 2012; Fischer et al., 2012). The bacteria are transmitted from animals to human by ingestion of infected food products (meat or raw milk), direct contact with infected animals or their tissues, or inhalation of aerosols. Humans are accidental hosts, but brucellosis continues to be a major public health concern worldwide and is the most widespread zoonotic infection.

Brucella genus does not exhibit classic virulence factors, such as exotoxins, exoproteases, cytolysins, or other exoenzymes (Moreno and Moriyon, 2002). Observed tissue harm is a result of inflammatory immune responses through the activation of host immune responses after recognition of brucellar antigens by immune receptors, such as Toll-like receptors (TLR) and inflammasomes (Campos et al., 2004, 2017; Giambartolomei et al., 2004; Zwerdling et al., 2008, 2009; García Samartino et al., 2010; de Almeida et al., 2011, 2013; Delpino et al., 2012; Gomes et al., 2013). Notwithstanding, with its intracellular lifestyle, Brucella limits exposure to innate and adaptive immune responses and leads the clinical manifestations of the disease and pathology. Brucella takes advantage of intracellular destruction by restringing fusion of Brucella-containing vacuoles with lysosomal compartments in a mechanism mediated by the type IV secretion system (T4SS) (Comerci et al., 2001). Also, Brucella inhibits the apoptosis of infected macrophages and prevents the development of adequate adaptive immune response by the inhibition of antigen presentation (de Figueiredo et al., 2015; Barrionuevo and Giambartolomei, 2019).

\section{CLINICAL FEATURES OF BRUCELLA LIVER INFECTION}

The liver is the most commonly affected organ in patients with active brucellosis. Accordingly, clinical and biochemical records of liver involvement have been observed in up to $50 \%$ of patients with active disease (Colmenero et al., 1996). Histopathological analyses of liver biopsies from a large number of patients with brucellosis have revealed liver parenchyma lesions due to inflammation, including focal areas of cellular inflammation with minimal necrosis of liver cells or the presence of granulomas with different localizations in parenchymal tissue and portal space (Akritidis et al., 2007). The pathology report on liver granulomas in Brucella infection usually shows necrotizing granulomas with a peripheral halo of epithelioid cells, lymphocytes, and plasma cells, as well as polymorphonuclear infiltrate in the necrotic area (Colmenero Jde et al., 2002; Villar et al., 2002). There are differences in the histological evaluation of liver manifestations in human brucellosis due to several causes. One of them is that most reports are retrospective and lack bacteriological confirmation. Also, previous reports with bacteriological confirmation did not always specify the species of Brucella involved. Moreover, not all of them used the same criteria to define granuloma (for example, presence of epithelioid cells or presence of giant cells) (Adams, 1976).

Liver biopsies also presented evidence of hepatitis (Akritidis et al., 2007). The liver conducts the metabolism of carbohydrates, proteins, and fats. Some of the enzymes and the end products of these metabolic pathways may be utilized as biochemical markers of liver dysfunction because they are very sensitive to any abnormality that takes place. Despite the abovementioned, the liver function is frequently normal in Brucella-infected individuals. The total serum bilirubin may be slightly increased, and the total serum protein, albumin, and globulin are usually normal. The most frequent abnormalities are shown by the increase in transaminases and alkaline phosphatase, although they are non-specific (Young, 1995; Madkour, 2001). In these patients, the presence of inflammatory infiltrates is also common, and most of them present parenchymal necrosis (Madkour, 2001; Akritidis et al., 2007).

\section{THE LIVER AS AN IMMUNE ORGAN}

The liver is usually regarded only as a non-immunological organ involved mainly in metabolic, nutrient storage, and detoxification activities. However, it is a member of the immune system responsible for the production of acute-phase proteins, chemokines, cytokines, complement proteins, and it also carries diverse populations of resident immune cells (O'Farrelly and Crispe, 1999; Crispe, 2009; Nemeth et al., 2009). Notwithstanding, the spleen is the main critical mediator in the clearance of blood pathogens, which overlaps with the function of the liver (Robinson et al., 2016).

Lymphocyte populations are present in the parenchyma and the portal tracts of the liver. These populations consist of conventional and unconventional lymphocytes of innate immunity, NKT and NK cells, as well as cells of the adaptive immune system, T and B cells (Freitas-Lopes et al., 2017). Resident antigen-presenting cells are also abundant in the liver. These cells are able to capture antigens that enter through the liver or are released by dead or infected hepatocytes. The group of resident antigen-presenting cells comprises Kupffer cells (members of the reticuloendothelial system) (Gale et al., 1978), liver sinusoidal endothelial cells (LSEC) (a particular type of vascular endothelial cells) (Steffan et al., 1986), and dendritic cells (DC) (Prickett et al., 1988; Lau and Thomson, 2003). The presence of these cells is essential for the maintenance of liver tolerance in physiological conditions (Robinson et al., 2016).

Kupffer cells are the liver resident macrophages that adhere to sinusoidal endothelial cells inside the sinusoids (Smith, 2013). This localization is adequate to perform its function as a scavenger removing protein complexes, small particles, senescent red blood cells, and cell debris from the portal blood via pattern recognition receptors (PRRs) (Petrasek et al., 2012).

LSEC are specialized endothelial cells that by the absence of diaphragm and lack of basement membrane are the most permeable endothelial cells in the body. In physiological conditions, these cells maintain hepatic stellate cell (HSC) 
quiescence inhibiting fibrosis development. These cells have a high phagocytic capacity and the molecules that promote antigen processing and presentation with efficacy similar to that of DC (Steffan et al., 1986; Lohse et al., 1996; Knolle et al., 1999).

Resident hepatic DC are located around the central veins and portal tracts. The presence of interleukin (IL)-10 and transforming growth factor (TGF) $\beta$ secreted by Kupffer and LSEC cells in the absence of infection provides a microenvironment capable of generating tolerant resident DC. The activation of these cells increases their capacity to migrate via the space of Disse to the lymphatic vessels in the portal tracts and then to the extrahepatic lymph nodes (Matsuno et al., 1996; Kudo et al., 1997).

Additionally, HSC and hepatocytes contribute to the immune homeostasis of the liver. Quiescent HSC exert immunoregulatory roles secreting chemokines, chemokine receptors, macrophage inflammatory proteins (MIPs), and TLR and also function as antigen-presenting cells (Friedman, 2008; Hernandez-Gea and Friedman, 2011). Hepatocytes can also participate as antigenpresenting cells, but they do not express the costimulatory molecules CD80 and CD86 (Bertolino et al., 1998). Therefore, hepatocytes induce T-cell functional activation but fail to promote survival (Bertolino et al., 1998). In addition, hepatocytes can also express PD-L1 in response to interferon (IFN) of type I and II with the consequent induction of T-cell apoptosis contributing to liver tolerance (Mühlbauer et al., 2006). This immunotolerant capacity of the liver is due to its structure, with resident immune cells in constant stimulation and the hepatic blood supply that creates a unique cytokine and growth factor milieu. This microenvironment determines the balance between tolerance and inflammation in the healthy liver. The complex of cytokine milieu in adult liver, in the absence of pathological inflammation, includes basal expression of proinflammatory cytokines IL-15, IL-7, IL-2, IL-12, IFN- $\gamma$, and the anti-inflammatory cytokines IL-10, IL- 13 , and TFG- $\beta$ (Golden-Mason et al., 2004; Kelly et al., 2006). The tolerogenic environment is maintained by regulatory myeloid populations, such as myeloid-derived suppressor cells (MDSC), that mediate their suppressive activity through the production of IL-10 and TGF- $\beta$ (Gabrilovich and Nagaraj, 2009). The transmigration of monocytes leads to MDSC differentiation and activation, contributing to the immunoregulatory capacity of the liver (Sander et al., 2010; Zimmermann et al., 2015). In particular, the impact of IL-10 in Brucella persistence and establishment of chronic infection through the modulation of macrophages has been demonstrated previously by using IL-10-deficient mice (Xavier et al., 2013).

Nevertheless, this immune tolerance, the hepatic immune system is able to induce rapid and controlled responses to pathogenic microorganisms and tumor cells (Robinson et al., 2016). Accordingly, most of the microorganisms that reach the liver through the blood are eliminated. Although the liver has several mechanisms to resist and to eliminate infectious agents, some of them, such as Brucella spp, take advantage of the immunotolerant capacity of the liver to escape from the immune response and persist in the host. Besides, because the liver is the organ with the largest number of resident macrophages
(Heymann and Tacke, 2016), we can speculate that the liver constitutes a place for Brucella persistence.

\section{BRUCELLA AND HSC IN FIBROSIS}

HSC reside in the liver between the hepatocytes and the small blood vessels. These cells are characterized as containing intracellular lipid droplets and protrusions that extend around the blood vessels. During liver damage, these cells are activated and secrete collagen with the formation of scar tissue, leading to chronic fibrosis or cirrhosis (Xu et al., 2012).

Brucella is an infectious stimulus that causes HSC activation that involves the conversion of quiescent cells into myofibroblasts, as revealed by the increase in $\alpha$-smooth muscle actin (SMA) expression, the increase in collagen secretion, the inhibition of matrix metalloproteinase (MMP)-9 secretion, the induction of the tissue inhibitor of metalloproteinases (TIMP)-1, and the secretion of the master regulator of fibrosis TGF- $\beta$ (Arriola Benitez et al., 2013). TGF- $\beta$ was identified as a main driver of HSC activation, and several approaches targeting the TGF- $\beta$ signaling pathway were successfully used to engage fibrosis in animal models of chronic liver diseases (Dooley and ten Dijke, 2012; Puche et al., 2013).

HSC secrete the chemokines IL-8 and the monocyte chemotactic protein (MCP)-1 in response to Brucella abortus infection that could attract to the site of infection, neutrophils and monocytes, respectively (Arriola Benitez et al., 2013). It has been demonstrated that $B$. abortus-infected monocytes could not reverse the fibrotic phenotype in B. abortus-infected HSC. However, B. abortus-infected monocytes inhibit collagen deposition and induce MMP-9 secretion in uninfected HSC (Arriola Benitez et al., 2013). This may explain, at least in part, why many patients with brucellosis have an inflammatory infiltrate, but only some of them develop cirrhosis. Moreover, in patients with brucellosis and cirrhosis, other causes such as viral hepatitis or alcoholic cirrhosis, have not been ruled out (Madkour, 2001).

Until now, it is unclear that Brucella infection can directly or indirectly modulate HSC activity and, consequently, the deposition of extracellular matrix. As in other liver diseases, HSC activation during B. abortus infection could contribute to granuloma formation by laying down a ring of collagen to encapsulate the granuloma (Chuah et al., 2014) (Figure 1).

\section{BRUCELLA TYPE IV SECRETION SYSTEM IN FIBROSIS}

T4SSs are a multiprotein complex involved in the translocation of nucleoproteins and/or protein substrates across the bacterial cell envelope to the host cell (Zechner et al., 2012). In Brucella, T4SS is encoded by the virB operon, which consists of 12 genes (virB1-12) located on chromosome II. The transcription of the virB operon is controlled by the promoter upstream of virB1 (O'Callaghan et al., 1999; Sieira et al., 2000). T4SS protein substrates have been shown to modulate several cellular processes in the host cell, such as apoptosis, vesicle trafficking, ubiquitination, and 


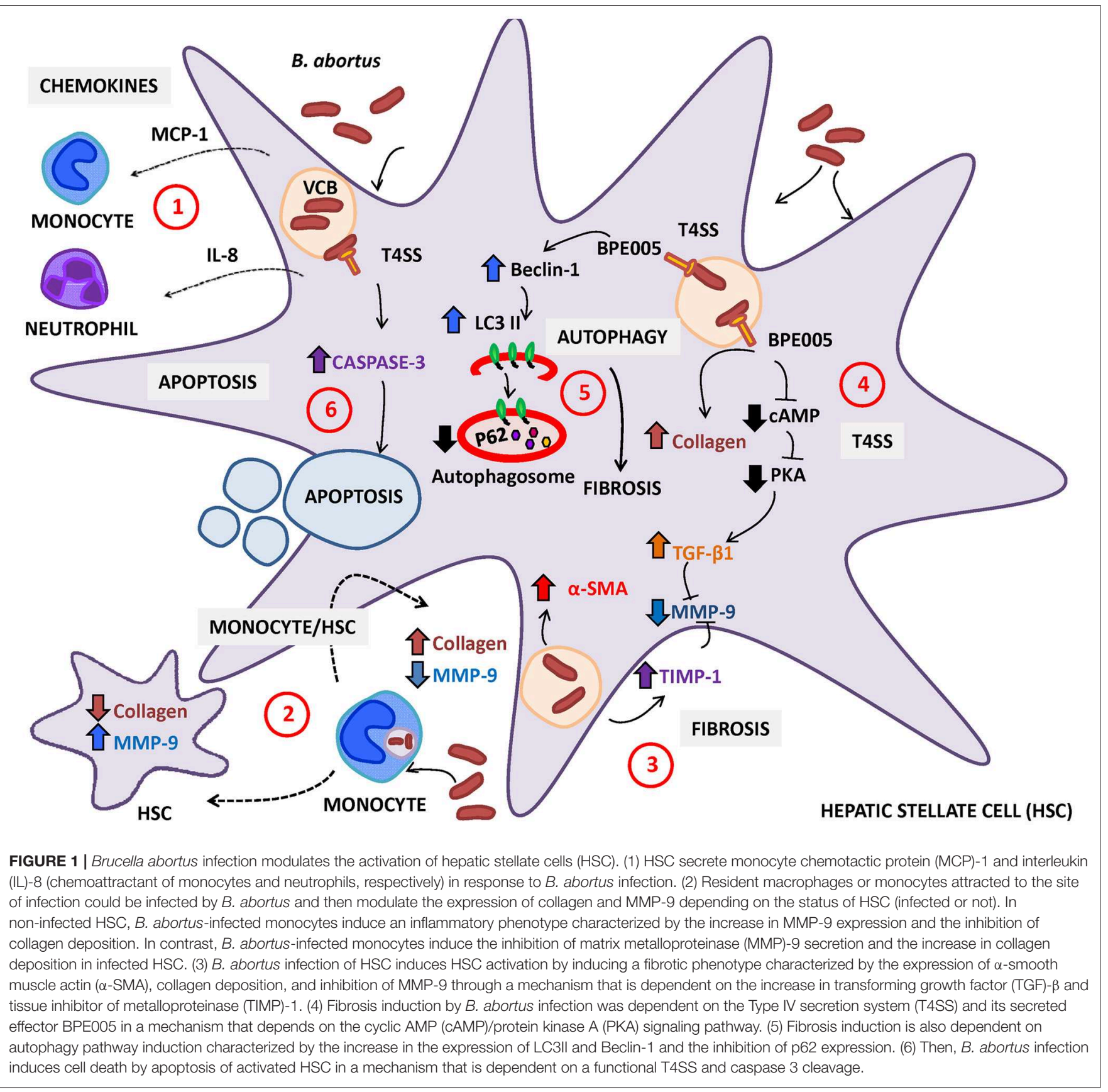

so on (Ninio and Roy, 2007; Franco et al., 2009). In Brucella, T4SS has been shown to be involved in the modulation of the immune system during infection (Roux et al., 2007; Rolán and Tsolis, 2008; Gomes et al., 2013); Brucella protein effector (BPE)005 is particularly involved in liver fibrosis (Arriola Benitez et al., 2016). The predicted structure BPE005 suggests that it might have an effect on cyclic AMP (cAMP) signaling pathways (Marchesini et al., 2011). In the HSC, BPE005 may mediate its effect by blocking the binding between cAMP and protein kinase A (PKA) with the concomitant fibrosis induction, as demonstrated in studies performed in vitro (Arriola Benitez et al.,
2016). Interestingly, the role of BPE005 in the modulation of the fibrotic phenotype during $B$. abortus infection was confirmed in an in vivo model in mice infected with a B. abortus BPE005 mutant. The histological analysis by Masson's trichrome staining revealed that the level of fibrotic patches is lower in mice infected with BPE005 mutants than in those infected with the wild-type counterpart. Accordingly, levels of collagen and TGF$\beta$ were lower in mice infected with the B. abortus BPE005 mutant (Arriola Benitez et al., 2016).

The role of the cAMP/PKA signaling pathway in the liver has been reported previously. The involvement of this 
pathway was demonstrated in various metabolic functions with the main effect in the facilitation of carbohydrate and lipid metabolism. cAMP plays a major role in the modulation of the HSC function by inhibiting profibrogenic pathways in HSC (Lopez-Sanchez et al., 2014).

Autophagy is involved in the fibrotic response during chronic hepatic lesion caused by hepatitis virus infection, alcohol abuse, and nonalcoholic steatohepatitis (Song et al., 2014).

Autophagy is a catabolic intracellular pathway that targets defective or excessive organelles to the lysosomes for degradation into amino acids, free fatty acids, or other small molecules used for material recycling or energy harvesting (Mao and Fan, 2015). Autophagy, usually stimulated by energy restriction, stress, or inflammation, is regarded as a survival mechanism that plays a critical role in maintaining cellular homeostasis, which is involved in many human disorders, such as fibrotic disease (Yin et al., 2008). In fibrosis, autophagy is mostly a cell survival mechanism that attenuates hepatic inflammatory injury and ultimately induces liver fibrosis (Mao and Fan, 2015). In addition, and supporting the role of BPE005 in the induction of HSC activation to a fibrotic phenotype, it has been demonstrated that autophagy is involved in the fibrotic response due to B. abortus, which depends on a functional T4SS and its effector BPE005 (Arriola Benitez et al., 2018). Autophagy was revealed by the upregulation of the LC3II/LC3I ratio and Beclin-1 expression and by the inhibition of p62 expression in infected cells (Arriola Benitez et al., 2018). However, further studies are necessary to determine if BPE005 could be a therapeutic target because this factor induces a fibrogenic phenotype that could help not only the host in the response to the inflammatory damage but also in the formation of granulomas likely favoring the persistence of bacterium.

Of note, the presence of liver cirrhosis during hepatic brucellosis is a debatable issue (Madkour, 2001). In addition, in murine models, cirrhosis was not observed upon infection with B. abortus (Arriola Benitez et al., 2013). In line with previous observations, $B$. abortus infection induces the clearance of activated HSC by apoptosis through a mechanism that is dependent on a functional T4SS and involved caspase 3 cleavage, triggering the recovery of liver fibrosis (Arriola Benitez et al., 2018) (Figure 1).

\section{BRUCELLA AND HEPATOCYTES}

Hepatocytes are the main cells of the liver parenchyma tissue and occupy around $70-85 \%$ of the liver volume (Kmieć, 2001). Hepatocytes are known to be involved in the synthesis of proteins, glycoproteins, cholesterol, bile salts, and phospholipids. On the other hand, they contribute to the detoxification and excretion of substances. However, these cells also participate in the immune response against pathogens. Hepatocytes respond to viral, bacterial, and parasitic infections by secreting proinflammatory cytokines and chemokines as mentioned elsewhere (Santos et al., 2005; McCord et al., 2006; Costa et al., 2008; Heydtmann and Adams, 2009), and B. abortus is not the exception. B. abortus infects hepatocytes and induces the secretion of IL-8, the main chemoattractant for neutrophil (Delpino et al., 2010), which correlates with the neutrophil infiltration observed in the liver of Brucella-infected patients (Hunt and Bothwell, 1967; Colmenero Jde et al., 2002). B. abortus infection also induces intercellular adhesion molecule (ICAM1) and MMP-9 secretion by hepatocytes (Delpino et al., 2010). These molecules ensure the influx of neutrophils to the tissues. ICAM-1 could facilitate the interaction of hepatocytes with neutrophils, and MMP-9 promotes neutrophil transmigration through degradation of extracellular matrix. These neutrophils could be also infected by $B$. abortus, and in response to this infection, they can contribute to the inflammatory reaction through the secretion of IL-8 and MMP-9 by inducing the expression of ICAM-1 by hepatocytes (Delpino et al., 2010).

Presence of liver parenchymal necrosis has been demonstrated in patients with hepatic manifestations of brucellosis (Akritidis et al., 2007), and hepatocytes occupy most of the parenchymal tissue. The analysis of the effects of $B$. abortus infection on hepatocyte viability revealed the induction of cell death by apoptosis and cytotoxic release of lactate dehydrogenase (LDH) in a T4SS-dependent manner (Delpino et al., 2010). Neutrophils also contribute to the induction of apoptosis and $\mathrm{LDH}$ release by hepatocytes as revealed by the stimulation with culture supernatants from $B$. abortus-infected neutrophils (Delpino et al., 2010).

B. abortus-infected hepatocytes can also modulate HSC function. Supernatants from B. abortus-infected hepatocytes induce MMP-9 secretion and inhibit collagen deposition in LX2 cells (Arriola Benitez et al., 2013). However, when stimulation was performed on B. abortus-infected HSC, supernatants from B. abortus-infected hepatocytes were unable to reverse the inhibitory effect of B. abortus infection in the inhibition of MMP9 secretion and the induction of collagen deposition on HSC (Arriola Benitez et al., 2013). The status of HSC (infected or not) defines if $B$. abortus-infected hepatocytes induce a fibrotic or an inflammatory phenotype in these cells (Figure 2).

\section{IN VIVO STUDIES FOR LIVER BRUCELLOSIS}

The absence of a suitable animal model that can reproduce, after experimental infection, the variety of disease manifestations of human brucellosis has determined the slow progress in most of the pathobiology of focal forms of the disease. However, while laboratory rodents do not mimic all of the spectrum of clinical signs and symptoms of human disease, there are focal manifestations of liver brucellosis (Olsen and Palmer, 2014). This allows characterizing at least in part, the pathophysiological and immune manifestations of hepatic brucellosis.

Studies performed in mice infected with Brucella melitensis stated that most of the infected cells in the liver expressed F4/80 myeloid marker. In the peak of infection, granuloma formation occurs. Granulomas are mainly composed by CD11b+ F4/80+ MHC-II+ cells expressing iNOS/NOS2 enzyme (Copin et al., 2012). A population of these cells also expressed 


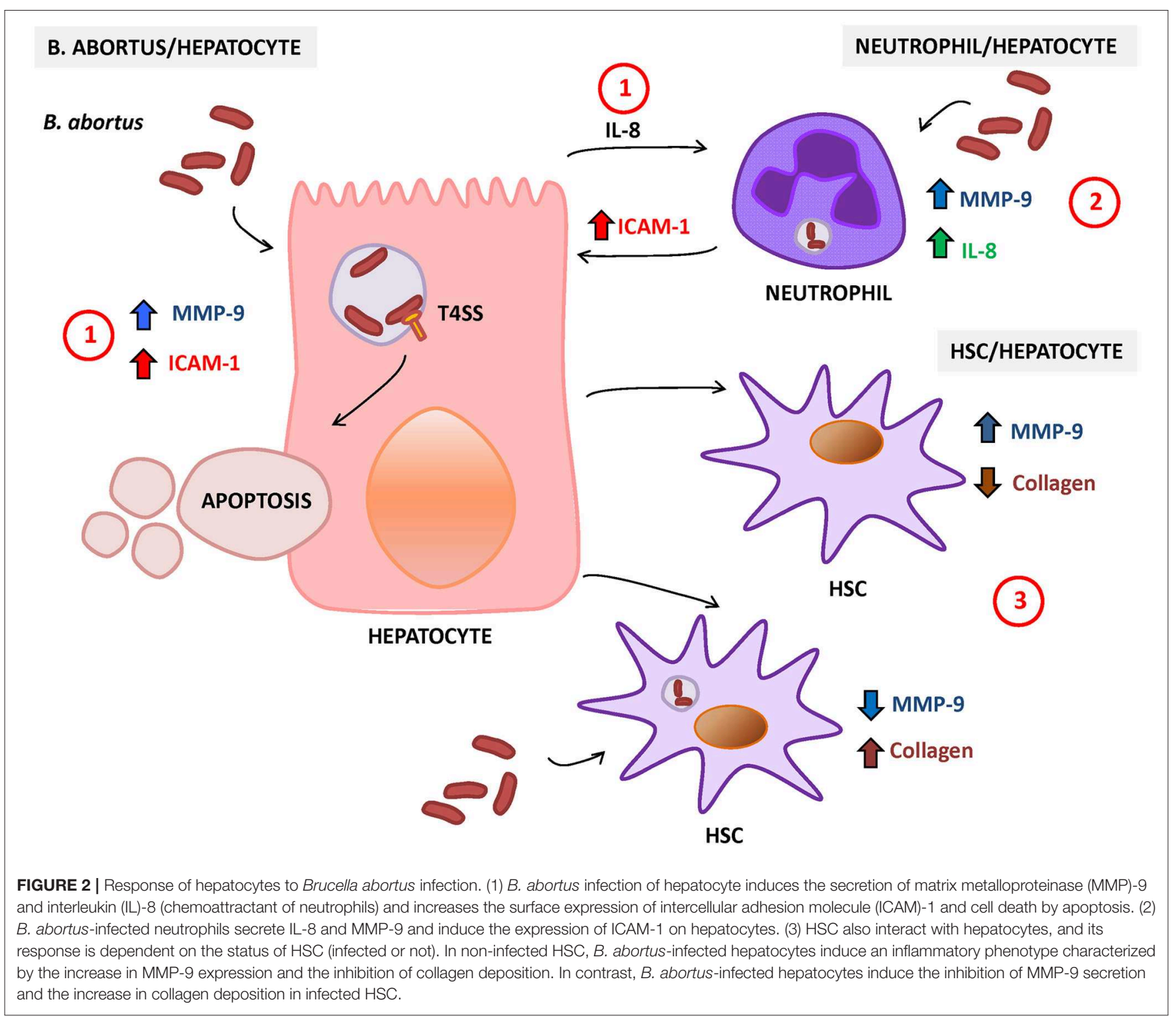

CD11c marker, indicating the presence of inflammatory DC. However, further analyses are necessary to determine if Brucella will remain in these cells or will migrate to others that protect them from the immune response (Copin et al., 2012). The delay in conducting these studies is due to the fact that the murine model does not represent all of the clinical manifestations of the disease, and that the liver biopsy is not a medical practice for the diagnosis of brucellosis.

In vivo studies also revealed that IL-10 production by $\mathrm{CD} 25^{+} \mathrm{CD} 4^{+} \mathrm{T}$ cells that modulate macrophage function enhances bacterial survival and persistence in liver through the modulation of the balance between pro-inflammatory and anti-inflammatory cytokines (Xavier et al., 2013). In addition, liver histopatology from infected $\mathrm{IL}-10^{-} /^{-}$mice at 3 weeks revealed multifocal granulomas and liver necrosis, as occurs in wild-type mice. However, at 6 weeks postinfection, the number of granulomas was reduced in $\mathrm{IL}-10^{-} /^{-}$mice with respect to wild-type controls. This reduction in liver pathology was accompanied by the increase of $\mathrm{CD} 4{ }^{+} \mathrm{CD} 25^{+}$foxp $3^{+} \mathrm{T}$ cells with the expression of TGF- $\beta$ (Corsetti et al., 2013). We can speculate that this reduction in liver pathology despite the increase in TGF$\beta$ can be attributed to the reduction of bacterial number and the consequent reduction in liver damage. However, further studies would be necessary to determine if the TGF- $\beta$ levels produced are capable of causing liver fibrosis in these mice.

In addition, intraperitoneal infection of mice with B. abortus allows corroborating in vitro findings, among them the role of BPE005 in the induction of liver fibrosis revealed by the increase in collagen deposition in liver and the expression of TGF- $\beta$ in HSC, as well as the presence of lymphocytic inflammatory infiltrate (Arriola Benitez et al., 2013, 2016). 
Despite the limitations of the rodent model, it allowed to corroborate the most relevant in vitro findings related to human clinical outcomes.

\section{CONCLUDING REMARKS}

Liver involvement in active brucellosis has ranged from 5 to $52 \%$ or more (Madkour, 2001). While the clinical and imaging aspect of hepatic brucellosis has been widely described, the molecular and cellular mechanisms involved in the damage and the immune response in the liver have only been partially clarified over the past 10 years. The findings summarized in this review attempt to answer the key questions about the soluble mediators, local and infiltrating cells involved in inflammatory damage during liver brucellosis.

The infection through the T4SS effector, BPE005, could modulate HSC activation to control the fibrotic process. It is likely that BPE005 could participate in granuloma formation that might act as a reservoir of bacteria contributing to the disease chronicity. Additionally, and in line with the presence of liver

\section{REFERENCES}

Adams, D. O. (1976). The granulomatous inflammatory response. A review. Am. J. Pathol. 84, 164-192.

Akritidis, N., Tzivras, M., Delladetsima, I., Stefanaki, S., Moutsopoulos, H. M., and Pappas, G. (2007). The liver in brucellosis. Clin. Gastroenterol. Hepatol. 5, 1109-1112. doi: 10.1016/j.cgh.2006.08.010

Arriola Benitez, P. C., Pesce Viglietti, A. I., Herrmann, C. K., Dennis, V. A., Comerci, D. J., Giambartolomei, G. H., et al. (2018). Brucella abortus promotes a fibrotic phenotype in hepatic stellate cells, with concomitant activation of the autophagy pathway. Infect. Immun. 86, e00522-17. doi: 10.1128/IAI.00522-17

Arriola Benitez, P. C., Rey Serantes, D., Herrmann, C. K., Pesce Viglietti, A. I., Vanzulli, S., Giambartolomei, G. H., et al. (2016). The effector protein BPE005 from Brucella abortus induces collagen deposition and matrix metalloproteinase 9 downmodulation via transforming growth factor betal in hepatic stellate cells. Infect. Immun. 84, 598-606. doi: 10.1128/IAI.01227-15

Arriola Benitez, P. C., Scian, R., Comerci, D. J., Serantes, D. R., Vanzulli, S., Fossati, C. A., et al. (2013). Brucella abortus induces collagen deposition and MMP9 down-modulation in hepatic stellate cells via TGF-betal production. Am. J. Pathol. 183, 1918-1927. doi: 10.1016/j.ajpath.2013.08.006

Barrionuevo, P., and Giambartolomei, G. H. (2019). Inhibition of antigen presentation by Brucella: many more than many ways. Microbes Infect. 21, 136-142. doi: 10.1016/j.micinf.2018.12.004

Bertolino, P., Trescol-Biémont, M. C., and Rabourdin-Combe, C. (1998). Hepatocytes induce functional activation of naive CD8+ T lymphocytes but fail to promote survival. Eur. J. Immunol. 28, 221-36.

Campos, M. A., Rosinha, G. M., Almeida, I. C., Salgueiro, X. S., Jarvis, B. W., Splitter, G. A., et al. (2004). Role of Toll-like receptor 4 in induction of cellmediated immunity and resistance to Brucella abortus infection in mice. Infect. Immun. 72, 176-186. doi: 10.1128/IAI.72.1.176-186.2004

Campos, P. C., Gomes, M. T., Guimarães, E. S., Guimarães, G., and Oliveira, S. C. (2017). TLR7 and TLR3 sense Brucella abortus RNA to induce proinflammatory cytokine production but they are dispensable for host control of infection. Front. Immunol. 8:28. doi: 10.3389/fimmu.2017.00028

Chuah, C., Jones, M. K., Burke, M. L., McManus, D. P., and Gobert, G. N. (2014). Cellular and chemokine-mediated regulation in schistosome-induced hepatic pathology. Trends Parasitol. 30, 141-150. doi: 10.1016/j.pt.2013.12.009

Colmenero Jde, D., Queipo-Ortuño, M. I., Maria Reguera, J., Angel SuarezMuñoz, M., Martín-Carballino, S., and Morata, P. (2002). Chronic hepatosplenic abscesses in Brucellosis. Clinico-therapeutic features and parenchymal necrosis observed in patients (Akritidis et al., 2007), $B$. abortus induces cell death of hepatocytes in a mechanism that also depends on a functional T4SS (Arriola Benitez et al., 2018). Altogether, this knowledge could frame the first approach toward the discovery of a new therapeutic target leading to new treatments that could be coadministrated with antibiotics aimed at reducing liver damage.

\section{AUTHOR CONTRIBUTIONS}

GG helped draft and correct the manuscript. MD drafted the manuscript.

\section{FUNDING}

This work was supported by grants PICT 2014-1111 and PICT 2015-0316 from Agencia Nacional of Promoción Científica y Tecnológica (ANPCYT, Argentina), by grant PIP 2015-20170200 from Consejo Nacional de Investigaciones científicas y técnicas (CONICET). molecular diagnostic approach. Diagn. Microbiol. Infect. Dis. 42, 159-167. doi: 10.1016/S0732-8893(01)00344-3

Colmenero, J. D., Reguera, J. M., Martos, F., Sánchez-De-Mora, D., Delgado, M., Causse, M., et al. (1996). Complications associated with Brucella melitensis infection: a study of 530 cases. Medicine 75, 195-211. doi: 10.1097/00005792-199607000-00003

Comerci, D. J., Martínez-Lorenzo, M. J., Sieira, R., Gorvel, J. P., and Ugalde, R. A. (2001). Essential role of the VirB machinery in the maturation of the Brucella abortus-containing vacuole. Cell. Microbiol. 3, 159-168. doi: 10.1046/j.1462-5822.2001.00102.x

Copin, R., Vitry, M. A., Hanot Mambres, D., Machelart, A., De Trez, C., Vanderwinden, J. M., et al. (2012). In situ microscopy analysis reveals local innate immune response developed around Brucella infected cells in resistant and susceptible mice. PLoS Pathog. 8:e1002575. doi: 10.1371/journal.ppat.1002575

Corsetti, P. P., de Almeida, L. A., Carvalho, N. B., Azevedo, V., Silva, T. M., Teixeira, H. C., et al. (2013). Lack of endogenous IL-10 enhances production of proinflammatory cytokines and leads to Brucella abortus clearance in mice. PLoS ONE 8:e74729. doi: 10.1371/journal.pone.0074729

Costa, J. D., Nogueira de Melo, A. C., Vermelho, A. B., Meirelles Mde, N., and Porrozzi, R. (2008). In vitro evidence for metallopeptidase participation in hepatocyte damage induced by Leishmania chagasi-infected macrophages. Acta Trop. 106, 175-183. doi: 10.1016/j.actatropica.2008.03.006

Crispe, I. N. (2009). The liver as a lymphoid organ. Annu. Rev. Immunol. 27, 147-163. doi: 10.1146/annurev.immunol.021908.132629

Cross, A. R., Baldwin, V. M., Roy, S., Essex-Lopresti, A. E., Prior, J. L., and Harmer, N. J. (2019). Zoonoses under our noses. Microbes Infect. 21, 10-19. doi: 10.1016/j.micinf.2018.06.001

de Almeida, L. A., Carvalho, N. B., Oliveira, F. S., Lacerda, T. L., Vasconcelos, A. C., Nogueira, L., et al. (2011). MyD88 and STING signaling pathways are required for IRF3-mediated IFN-beta induction in response to Brucella abortus infection. PLoS ONE 6:e23135. doi: 10.1371/journal.pone.0023135

de Almeida, L. A., Macedo, G. C., Marinho, F. A., Gomes, M. T., Corsetti, P. P., Silva, A. M., et al. (2013). Toll-like receptor 6 plays an important role in host innate resistance to Brucella abortus infection in mice. Infect. Immun. 81, 1654-1662. doi: 10.1128/IAI.01356-12

de Figueiredo, P., Ficht, T. A., Rice-Ficht, A., Rossetti, C. A., and Adams, L. G. (2015). Pathogenesis and immunobiology of brucellosis: review of Brucella-host interactions. Am. J. Pathol. 185, 1505-1517. doi: 10.1016/j.ajpath.2015.03.003 
Delpino, M. V., Barrionuevo, P., Macedo, G. C., Oliveira, S. C., Genaro, S. D., Scian, R., et al. (2012). Macrophage-elicited osteoclastogenesis in response to Brucella abortus infection requires TLR2/MyD88-dependent TNF-alpha production. J. Leukoc. Biol. 91, 285-298. doi: 10.1189/jlb.04111185

Delpino, M. V., Barrionuevo, P., Scian, R., Fossati, C. A., and Baldi, P. C. (2010). Brucella-infected hepatocytes mediate potentially tissue-damaging immune responses. J. Hepatol. 53, 145-154. doi: 10.1016/j.jhep.2010.02.028

Dooley, S., and ten Dijke, P. (2012). TGF-beta in progression of liver disease. Cell Tissue Res. 347, 245-256. doi: 10.1007/s00441-011-1246-y

Eisenberg, T., Hamann, H. P., Kaim, U., Schlez, K., Seeger, H., Schauerte, N., et al. (2012). Isolation of potentially novel Brucella spp. from frogs. Appl. Environ. Microbiol. 78, 3753-3755. doi: 10.1128/AEM.07509-11

Fischer, D., Lorenz, N., Heuser, W., Kämpfer, P., Scholz, H. C., and Lierz, M. (2012). Abscesses associated with a Brucella inopinata-like bacterium in a big-eyed tree frog (Leptopelis vermiculatus). J. Zoo Wildl. Med. 43, 625-628. doi: 10.1638/2011-0005R2.1

Franco, I. S., Shuman, H. A., and Charpentier, X. (2009). The perplexing functions and surprising origins of Legionella pneumophila type IV secretion effectors. Cell. Microbiol. 11, 1435-1443. doi: 10.1111/j.1462-5822.2009.01351.x

Freitas-Lopes, M. A., Mafra, K., David, B. A., Carvalho-Gontijo, R., and Menezes, G. B. (2017). Differential location and distribution of hepatic immune cells. Cells 6:E48. doi: 10.3390/cells6040048

Friedman, S. L. (2008). Hepatic stellate cells: protean, multifunctional, and enigmatic cells of the liver. Physiol. Rev. 88, 125-172. doi: 10.1152/physrev.00013.2007

Gabrilovich, D. I., and Nagaraj, S. (2009). Myeloid-derived suppressor cells as regulators of the immune system. Nat. Rev. Immunol. 9, 162-174. doi: $10.1038 /$ nri2506

Gale, R. P., Sparkes, R. S., and Golde, D. W. (1978). Bone marrow origin of hepatic macrophages (Kupffer cells) in humans. Science 201, 937-938. doi: $10.1126 /$ science. 356266

García Samartino, C., Delpino, M. V., Pott Godoy, C., Di Genaro, M. S., Pasquevich, K. A., Zwerdling, A., et al. (2010). Brucella abortus induces the secretion of proinflammatory mediators from glial cells leading to astrocyte apoptosis. Am. J. Pathol. 176, 1323-1338. doi: 10.2353/ajpath.2010.090503

Giambartolomei, G. H., Zwerdling, A., Cassataro, J., Bruno, L., Fossati, C. A., and Philipp, M. T. (2004). Lipoproteins, not lipopolysaccharide, are the key mediators of the proinflammatory response elicited by heat-killed Brucella abortus. J. Immunol. 173, 4635-4642. doi: 10.4049/jimmunol.173.7.4635

Godfroid, J., Al Dahouk, S., Pappas, G., Roth, F., Matope, G., Muma, J., et al. (2013). A "One Health" surveillance and control of brucellosis in developing countries: moving away from improvisation. Comp. Immunol. Microbiol. Infect. Dis. 36, 241-248. doi: 10.1016/j.cimid.2012.09.001

Golden-Mason, L., Kelly, A. M., Doherty, D. G., Traynor, O., McEntee, G., Kelly, J., et al. (2004). Hepatic interleuklin 15 (IL-15) expression: implications for local NK/NKT cell homeostasis and development. Clin. Exp. Immunol. 138, 94-101. doi: 10.1111/j.1365-2249.2004.02586.x

Gomes, M. T., Campos, P. C., Oliveira, F. S., Corsetti, P. P., Bortoluci, K. R., Cunha, L. D., et al. (2013). Critical role of ASC inflammasomes and bacterial type IV secretion system in caspase- 1 activation and host innate resistance to Brucella abortus infection. J. Immunol. 190, 3629-3638. doi: 10.4049/jimmunol.1202817

Hernandez-Gea, V., and Friedman, S. L. (2011). Pathogenesis of liver fibrosis. Annu. Rev. Pathol. 6, 425-456. doi: 10.1146/annurev-pathol-011110-130246

Heydtmann, M., and Adams, D. H. (2009). Chemokines in the immunopathogenesis of hepatitis C infection. Hepatology 49, 676-688. doi: 10.1002/hep. 22763

Heymann, F., and Tacke, F. (2016). Immunology in the liver-from homeostasis to disease. Nat. Rev. Gastroenterol. Hepatol. 13, 88-110. doi: $10.1038 /$ nrgastro.2015.200

Hull, N. C., and Schumaker, B. A. (2018). Comparisons of brucellosis between human and veterinary medicine. Infect. Ecol. Epidemiol. 8:1500846. doi: 10.1080/20008686.2018.1500846

Hunt, A. C., and Bothwell, P. W. (1967). Histological findings in human brucellosis. J. Clin. Pathol. 20, 267-272. doi: 10.1136/jcp.20.3.267

Kelly, A. M., Golden-Mason, L., Traynor, O., Geoghegan, J., McEntee, G., Hegarty, J. E., et al. (2006). Changes in hepatic immunoregulatory cytokines in patients with metastatic colorectal carcinoma: implications for hepatic anti-tumour immunity. Cytokine 35, 171-179. doi: 10.1016/j.cyto.2006.07.019
Kmieć, Z. (2001). Cooperation of liver cells in health and disease. Adv. Anat. Embryol. Cell Biol/ 161:III-XIII, 1-151. doi: 10.1007/978-3-642-56553-3

Knolle, P. A., Schmitt, E., Jin, S., Germann, T., Duchmann, R., Hegenbarth, S., et al. (1999). Induction of cytokine production in naive CD4(+) T cells by antigen-presenting murine liver sinusoidal endothelial cells but failure to induce differentiation toward Th1 cells. Gastroenterology 116, 1428-1440. doi: 10.1016/S0016-5085(99)70508-1

Kudo, S., Matsuno, K., Ezaki, T., and Ogawa, M. (1997). A novel migration pathway for rat dendritic cells from the blood: hepatic sinusoids-lymph translocation. $J$. Exp. Med. 185, 777-784. doi: 10.1084/jem.185.4.777

Lau, A. H., and Thomson, A. W. (2003). Dendritic cells and immune regulation in the liver. Gut 52, 307-314. doi: 10.1136/gut.52.2.307

Lohse, A. W., Knolle, P. A., Bilo, K., Uhrig, A., Waldmann, C., Ibe, M., et al. (1996). Antigen-presenting function and B7 expression of murine sinusoidal endothelial cells and Kupffer cells. Gastroenterology 110, 1175-1181. doi: 10.1053/gast.1996.v110.pm8613007

Lopez-Sanchez, I., Dunkel, Y., Roh, Y. S., Mittal, Y., De Minicis, S., Muranyi, A., et al. (2014). GIV/Girdin is a central hub for profibrogenic signalling networks during liver fibrosis. Nat. Commun. 5:4451. doi: 10.1038/ncomms5451

Madkour, M. M. (2001). "Gastrointestinal brucellosis," in Madkour's Brucellosis, 2nd ed, eds M. M. Madkour (Berlin: Springer-Verlag), 150-158. doi: 10.1007/978-3-642-59533-2_13

Mancini, F. R., Bella, A., Graziani, C., Marianelli, C., Mughini-Gras, L., Pasquali, P., et al. (2014). Trends of human brucellosis in Italy, 1998-2010. Epidemiol. Infect. 142, 1188-1195. doi: 10.1017/S0950268813002227

Mao, Y. Q., and Fan, X. M. (2015). Autophagy: a new therapeutic target for liver fibrosis. World J. Hepatol. 7, 1982-1986. doi: 10.4254/wjh.v7.i16.1982

Marchesini, M. I., Herrmann, C. K., Salcedo, S. P., Gorvel, J. P., and Comerci, D. J. (2011). In search of Brucella abortus type IV secretion substrates: screening and identification of four proteins translocated into host cells through VirB system. Cell. Microbiol. 13, 1261-1274. doi: 10.1111/j.1462-5822.2011.01618.x

Matsuno, K., Ezaki, T., Kudo, S., and Uehara, Y. (1996). A life stage of particleladen rat dendritic cells in vivo: their terminal division, active phagocytosis, and translocation from the liver to the draining lymph. J. Exp. Med. 183, 1865-1878. doi: 10.1084/jem.183.4.1865

McCord, A. M., Resto-Ruiz, S. I., and Anderson, B. E. (2006). Autocrine role for interleukin-8 in Bartonella henselae-induced angiogenesis. Infect. Immun. 74, 5185-5190. doi: 10.1128/IAI.00622-06

Moreno, E., and Moriyon, I. (2002). Brucella melitensis: a nasty bug with hidden credentials for virulence. Proc. Natl. Acad. Sci. U.S.A. 99, 1-3. doi: 10.1073/pnas.022622699

Mühlbauer, M., Fleck, M., Schütz, C., Weiss, T., Froh, M., Blank, C., et al. (2006). PD-L1 is induced in hepatocytes by viral infection and by interferonalpha and -gamma and mediates $\mathrm{T}$ cell apoptosis. J. Hepatol. 45, 520-528. doi: 10.1016/j.jhep.2006.05.007

Nemeth, E., Baird, A. W., and O'Farrelly, C. (2009). Microanatomy of the liver immune system. Semin. Immunopathol. 31, 333-343. doi: 10.1007/s00281-009-0173-4

Ninio, S., and Roy, C. R. (2007). Effector proteins translocated by Legionella pneumophila: strength in numbers. Trends Microbiol. 15, 372-380. doi: 10.1016/j.tim.2007.06.006

O’Callaghan, D., Cazevieille, C., Allardet-Servent, A., Boschiroli, M. L., Bourg, G., Foulongne, V., et al. (1999). A homologue of the Agrobacterium tumefaciens VirB and Bordetella pertussis Ptl type IV secretion systems is essential for intracellular survival of Brucella suis. Mol. Microbiol. 33, 1210-1220. doi: 10.1046/j.1365-2958.1999.01569.x

O'Farrelly, C., and Crispe, I. N. (1999). Prometheus through the looking glass: reflections on the hepatic immune system. Immunol. Today 20, 394-398. doi: 10.1016/S0167-5699(99)01518-2

Olsen, S. C., and Palmer, M. V. (2014). Advancement of knowledge of Brucella over the past 50 years. Vet. Pathol. 51, 1076-1089. doi: 10.1177/03009858145 40545

Pappas, G., Papadimitriou, P., Akritidis, N., Christou, L., and Tsianos, E. V. (2006). The new global map of human brucellosis. Lancet Infect. Dis. 6, 91-99. doi: 10.1016/S1473-3099(06)70382-6

Petrasek, J., Bala, S., Csak, T., Lippai, D., Kodys, K., Menashy, V., et al. (2012). IL-1 receptor antagonist ameliorates inflammasome-dependent alcoholic steatohepatitis in mice. J. Clin. Invest. 122, 3476-3489. doi: 10.1172/JCI60777 
Prickett, T. C., McKenzie, J. L., and Hart, D. N. (1988). Characterization of interstitial dendritic cells in human liver. Transplantation 46, 754-761. doi: 10.1097/00007890-198811000-00024

Puche, J. E., Lee, Y. A., Jiao, J., Aloman, C., Fiel, M. I., Muñoz, U., et al. (2013). A novel murine model to deplete hepatic stellate cells uncovers their role in amplifying liver damage in mice. Hepatology 57, 339-350. doi: 10.1002/hep.26053

Robinson, M. W., Harmon, C., and O'Farrelly, C. (2016). Liver immunology and its role in inflammation and homeostasis. Cell. Mol. Immunol. 13, 267-276. doi: $10.1038 / \mathrm{cmi} .2016 .3$

Rolán, H. G., and Tsolis, R. M. (2008). Inactivation of the type IV secretion system reduces the Th1 polarization of the immune response to Brucella abortus infection. Infect. Immun. 76, 3207-3213. doi: 10.1128/IAI.00203-08

Roux, C. M., Rolán, H. G., Santos, R. L., Beremand, P. D., Thomas, T. L., Adams, L. G., et al. (2007). Brucella requires a functional Type IV secretion system to elicit innate immune responses in mice. Cell. Microbiol. 9, 1851-1869. doi: 10.1111/j.1462-5822.2007.00922.x

Sander, L. E., Sackett, S. D., Dierssen, U., Beraza, N., Linke, R. P., Müller, M., et al. (2010). Hepatic acute-phase proteins control innate immune responses during infection by promoting myeloid-derived suppressor cell function. J. Exp. Med. 207, 1453-1464. doi: 10.1084/jem.20091474

Santos, S. A., Andrade, D. R., and Andrade Júnior, D. R. (2005). Rat hepatocyte invasion by Listeria monocytogenes and analysis of TNFalpha role in apoptosis. Rev. Inst. Med. Trop. São Paulo 47, 73-80. doi: 10.1590/S0036-46652005000200003

Sieira, R., Comerci, D. J., Sánchez, D. O., and Ugalde, R. A. (2000). A homologue of an operon required for DNA transfer in Agrobacterium is required in Brucella abortus for virulence and intracellular multiplication. J. Bacteriol. 182, 4849-4855. doi: 10.1128/JB.182.17.4849-485 5.2000

Smith, K. (2013). Liver disease: kupffer cells regulate the progression of ALD and NAFLD. Nat. Rev. Gastroenterol. Hepatol. 10:503. doi: 10.1038/nrgastro.2013.140

Song, Y., Zhao, Y., Wang, F., Tao, L., Xiao, J., and Yang, C. (2014). Autophagy in hepatic fibrosis. Biomed Res. Int. 2014:436242. doi: 10.1155/2014/4 36242

Steffan, A. M., Gendrault, J. L., McCuskey, R. S., McCuskey, P. A., and Kirn, A. (1986). Phagocytosis, an unrecognized property of murine endothelial liver cells. Hepatology 6, 830-836. doi: 10.1002/hep.18400 60505
Villar, J. M., Garrote, D., Villegas, M. T., Alvarez, M. J., Mansilla, A., and Ferrón, J. A. (2002). Hepatic brucelloma. J. Am. Coll. Surg. 194:86. doi: 10.1016/S1072-7515(01)01111-5

Xavier, M. N., Winter, M. G., Spees, A. M., Nguyen, K., Atluri, V. L., Silva, T. M., et al. (2013). CD4+ T cell-derived IL-10 promotes Brucella abortus persistence via modulation of macrophage function. PLoS Pathog. 9:e1003454. doi: 10.1371/journal.ppat.1003454

Xu, R., Zhang, Z., and Wang, F. S. (2012). Liver fibrosis: mechanisms of immunemediated liver injury. Cell. Mol. Immunol. 9, 296-301. doi: 10.1038/cmi.2011.53

Yin, X. M., Ding, W. X., and Gao, W. (2008). Autophagy in the liver. Hepatology 47, 1773-1785. doi: 10.1002/hep.22146

Young, E. J. (1995). An overview of human brucellosis. Clin. Infect. Dis. 21, 283-9; quiz 90. doi: 10.1093/clinids/21.2.283

Zechner, E. L., Lang, S., and Schildbach, J. F. (2012). Assembly and mechanisms of bacterial type IV secretion machines. Philos. Trans. R. Soc. Lond, B,. Biol. Sci. 367, 1073-1087. doi: 10.1098/rstb.2011.0207

Zimmermann, H. W., Bruns, T., Weston, C. J., Curbishley, S. M., Liaskou, E., Li, K. K., et al. (2015). Bidirectional transendothelial migration of monocytes across hepatic sinusoidal endothelium shapes monocyte differentiation and regulates the balance between immunity and tolerance in liver. Hepatology 63, 233-246. doi: 10.1002/hep.28285

Zwerdling, A., Delpino, M. V., Barrionuevo, P., Cassataro, J., Pasquevich, K. A., García Samartino, C., et al. (2008). Brucella lipoproteins mimic dendritic cell maturation induced by Brucella abortus. Microbes Infect. 10, 1346-1354. doi: 10.1016/j.micinf.2008.07.035

Zwerdling, A., Delpino, M. V., Pasquevich, K. A., Barrionuevo, P., Cassataro, J., García Samartino, C., et al. (2009). Brucella abortus activates human neutrophils. Microbes Infect. 11, 689-697. doi: 10.1016/j.micinf.2009.04.010

Conflict of Interest: The authors declare that the research was conducted in the absence of any commercial or financial relationships that could be construed as a potential conflict of interest.

Copyright (c) 2019 Giambartolomei and Delpino. This is an open-access article distributed under the terms of the Creative Commons Attribution License (CC BY). The use, distribution or reproduction in other forums is permitted, provided the original author(s) and the copyright owner(s) are credited and that the original publication in this journal is cited, in accordance with accepted academic practice. No use, distribution or reproduction is permitted which does not comply with these terms. 